\title{
Haftungskonzepte im römischen Deliktsrecht
}

\author{
Univ.-Prof. Dr. Cosima Möller, Freie Universität Berlin
}

\section{A. Einleitung-Zur Rolle einer historischen Skizze, mit einigen Bemerkungen zum Charakter des römischen Rechts}

Dieser Tagungsbeitrag verdankt seine Entstehung der rechtshistorischen Aufgeschlossenheit der Veranstalter. Dafür sei an dieser Stelle ausdrücklich und herzlich gedankt. Der thematische Hintergrund ist die Suche nach einer möglichst klaren Zuweisung von Verantwortung. Das besondere Augenmerk gilt dabei den »Intelligenten Agenten«, also den durch menschliche Intelligenz geschaffenen Akteuren, die als »Automaten« nicht nur selbst ein vorgesehenes Programm umsetzen, sondern durch die Auswertung von Daten im Rahmen einer Programmierung Verhaltensweisen an den Tag legen, die eine Subsumtionsleistung voraussetzen und damit Lernfähigkeit dokumentieren. ${ }^{1}$ Durch die Programmierung sind die für das Handeln wichtigen Informationen in einen Entscheidungsprozess eingeführt, der zu einem optimalen Funktionieren des »Automaten« führen soll. Wäre dieses Ziel erreichbar, müsste nicht über Fragen der Zurechnung gesprochen werden, weil die Störanfälligkeit menschlichen Handelns durch die Programmierung gerade ausgeräumt wäre.

Der Konjunktiv Irrealis zeigt an, dass die Tagung nicht überflüssig war. So wie das Recht mit seinen notwendig abstrakten Regelungen nicht jeden Einzelfall dergestalt zu erfassen vermag, dass eine Subsumtion als logische Operation »vorprogrammiert« zu einer gerechten Entscheidung führen würde, so wenig wird es einem Programm möglich sein, alle Faktoren zu berücksichtigen, die einen automatisierten Ablauf beeinflussen. ${ }^{2}$ Beim

1 Es geht also nicht um konventionelle Datenverarbeitung beim Einsatz von Computerprogrammen, die lediglich Aufgaben eines Auftraggebers ausführen, sondern um intelligente Agenten, die auf der Basis eigener Lernprozesse bzw. Wissenserkenntnisse Probleme eigenständig lösen, so St. Kirn/C. D. Müller-Hengstenberg, Intelligente (Software-)Agenten: Von der Automatisierung zur Autonomie? Verselbständigung technischer Systeme, MMR 2014, S. 225 (231 f.).

2 Unabhängig von der grundsätzlichen Ausrichtung der ,sozialen“ Fähigkeiten - man unterscheidet zwischen benevolent, kompetitiv, antagonistisch, strategisch und syn- 
Betrieb von Flugzeugen, von Eisenbahnen, Schiffen und Kraftfahrzeugen gibt es in unterschiedlicher Verbreitung »Autopiloten«. Doch ist das bewusste Abschalten dieser Systeme jedenfalls zurzeit möglich und wird als erforderlich angesehen. Es bleibt also die Verantwortlichkeit dessen erhalten, der sich dieser Systeme bedient. Bei einer vollständigen Selbststeuerung intelligenter Agenten, ohne dass menschliche Einflussnahme möglich ist, müssen die Überlegungen für eine Haftung an den Umstand anknüpfen, dass mit dem Einsatz eines solchen Agenten eine Gefahr bewusst in Kauf genommen worden ist oder - wie in der Wirtschaftsinformatik formuliert worden ist -, dass jemand diesen intelligenten Agenten »von der Leine gelassen hat . $^{3}$

Das grundlegende Thema menschlicher Verantwortlichkeit und ihrer Reichweite, das auch die strafrechtliche Diskussion leitet ${ }^{4}$, macht den Brückenschlag in die Geschichte zu einem wichtigen Hilfsmittel bei der Analyse der juristischen Probleme und bei ihrer Lösung. Der analytische Nutzen rechtsgeschichtlicher Betrachtung darf auf allgemeine Zustimmung hoffen. In welchem Maße die Heranziehung des römischen Rechts geeignet ist, dogmengeschichtliche Klarheit im Sinne einer historischen Entwicklungsgeschichte zu liefern, hängt entscheidend davon ab, ob man das römische Recht und die uns erhaltene Überlieferung desselben als Sammlung vielfältig interessanter Fallentscheidungen mit einem starken Sinn der römischen Juristen für praktische Lösungen ansieht ${ }^{5}$ oder in dieser Überlieferung konzeptionelle, systembegründende Zusammenhänge erkennt. ${ }^{6}$ Darüber sind die Meinungen unter den Römischrechtlern geteilt und auch zwischen diesen beiden Polen werden unterschiedliche Positionen eingenommen. ${ }^{7}$ Betrachtet man den sich daraus ergebenden Unter-

ergetisch - bleibe eine Kontrolle von Lern- und Problemlösungsfähigkeiten lückenhaft, wie Kirn und Müller-Hengstenberg (Fn. 1), S. 225 (229 f.) festhalten.

3 Kirn/Müller-Hengstenberg (Fn. 1), S. 225 (227).

4 Vgl. dazu eingehend S. Gleß/Th. Weigend, Intelligente Agenten und das Strafrecht, ZStW 126 (2014), S. 561-591.

5 So zum Beispiel Th. Finkenauer, D. 41,1,44: Das Schwein im Wolfsmaul, in: U. Falk, M. Luminati, M. Schmoeckel (Hrsg.), Fälle aus der Rechtsgeschichte, München 2008, S. 45 (60).

6 So insbesondere $O$. Behrends, Le due giurisprudenze romane e le forme delle loro argomentazioni, Index 12 (1983-84), S. 189-225. Weitere Hinweise s. Fn. 8.

7 Man beachte insoweit die Charakterisierung des römischen Rechts als offenes System bei R. Zimmermann, Römisches Recht und europäische Kultur, JZ 2007, S. 1 (7) oder auch die Formulierung, ,inneres System“ bei M. Kaser/R. Knütel, Römi- 
schied, so kann man in manchen Fällen sagen, dass die gegenüber konzeptionellen Entwürfen offene Betrachtungsweise eine weitere Interpretationsebene bietet und damit einen Schlüssel zu einem tiefergehenden Verständnis bereitstellt. Manchmal führt diese Einordnung aber auch zu anderen Erklärungsmustern. Dies betrifft zum Beispiel den historischen Ablauf der Streitigkeiten zwischen den römischen Juristen, ob man eine Entwicklungslinie nachzeichnen kann oder ob interpretatorische Einschnitte aufgrund neuer Konzepte festzustellen sind. Die Thematik ist für die römische Rechtsgeschichte nichts weniger als grundlegend. Doch für die Zwecke einer Unterstützung beim Nachdenken über aktuelle Rechtsfragen muss man zu dieser Charakteristik des römischen Rechts nicht notwendig Stellung beziehen. Dass ich die Annahme teile, die als erster Okko Behrends Ende der 70er Jahre des 20. Jhs. entwickelt und in zahlreichen Aufsätzen entfaltet hat, ${ }^{8}$ dass nämlich konzeptionelle Überlegungen in der republikanischen Zeit unter dem Einfluss der griechischen Philosophie die Diskussion im römischen Recht geprägt und auch in der Prinzipatszeit in den Rechtsschulen der Sabinianer und Prokulianer weitergewirkt haben, habe ich für die Grunddienstbarkeiten im römischen Recht, die Servituten, in meiner Habilitationsschrift entwickelt und auch in verschiedenen Aufsätzen für das Vertragsrecht und für das Deliktsrecht dargelegt. ${ }^{9}$ Die Grundannahmen haben sich seitdem auf den unterschiedlichsten Feldern

sches Privatrecht, 20. Aufl., München 2014, S. 30 f. W. Waldstein/J. M. Rainer, Römische Rechtsgeschichte, 11. Aufl., München 2014, S. 220 ff. sprechen von einem offenen, von der Sache bestimmten und nicht willkürlich konstruierten System, das sich durch die Ergebnisse der Arbeit der römischen Juristen ergeben habe, obwohl sie es selbst gar nicht anstrebten. Diese „stillwirkenden Kräfte“ erinnern an die historische Rechtsschule.

8 O. Behrends, Institutionelles und prinzipielles Denken im römischen Privatrecht, SZ 95 (1978), S. 187-231 (=Institut und Prinzip, Band 1, Göttingen 2004, S. 15-50) und insbesondere die Sammlung von Aufsätzen in den beiden Bänden O. Behrends, Institut und Prinzip, in: M. Avenarius, R. Meyer-Pritzl und C. Möller (Hrsg.), Göttingen 2004 sowie aus der jüngeren Zeit O. Behrends, Die geistige Mitte des römischen Rechts. Die Kulturanthropologie der skeptischen Akademie, SZ 125 (2008), S. 25-107 und ders., Wie haben wir uns die römischen Juristen vorzustellen?, SZ 128 (2011), S. 83-129.

9 C. Möller, Die Servituten. Entwicklungsgeschichte, Funktion und Struktur der grundstücksvermittelten Privatrechtsverhältnisse im römischen Recht. Mit einem Ausblick auf die Rezeptionsgeschichte und das BGB. Göttingen 2010; dies., Grundlagen des römischen Vertragsrechts, in: Philip Kunig, Makoto Nagata (Hrsg.), Deutschland und Japan im rechtswissenschaftlichen Dialog, Köln 2006, S. 71-90; dies., Via publica und via privata im römischen Deliktsrecht, in: M. Avenarius, 
bestätigt. Für die im Zusammenhang der Tagung zu behandelnde Frage lassen sich frühere Untersuchungen zum Deliktsrecht weiterführen.

Fragen der deliktischen Haftung sind im römischen Recht in vielfältiger Weise geregelt und unter den Juristen in zugleich konzeptionell anspruchsvoller wie am praktischen Fall orientierter Weise diskutiert und entschieden worden. Der Einsatz von Akteuren, die selbst nicht zu einer Haftung in der Lage sind, weil es ihnen am Vermögen fehlt - wie Hauskinder und Sklaven - oder bereits an der Zurechnungsfähigkeit - wie Schuldunfähigen oder Haustieren -, ist in einer besonderen Weise durch die Noxalhaftung geregelt gewesen. Zu diesem Thema informiert der Beitrag von Jan Dirk Harke in diesem Band. Aber auch die Möglichkeit des Haftenden, den schädigenden Akteur - vom Haussohn, über den Sklaven bis zum Haustier - an das Opfer auszuliefern anstatt die als Schadensersatz geschätzte Summe Geldes zu zahlen, knüpft notwendig an allgemeine Haftungskonzepte an. ${ }^{10}$ Auch hier muss der Schaden dem Akteur zugewiesen werden. ${ }^{11}$

Im Folgenden soll es um Konzepte deliktischer Haftung im römischen Recht gehen. In den historischen Abriss sind Überlegungen zu konzeptionellen Unterschieden und $\mathrm{zu}$ dogmatischen Potentialen eingefügt. Ein Schwerpunkt liegt auf Erläuterungen zur lex Aquilia, einem Beispiel von Gefährdungshaftung und der Bewertung eines Unfallgeschehens mit Maultieren. Schlussfolgerungen runden den Beitrag ab und schlagen den Bogen zur aktuellen Thematik.

R. Meyer-Pritzl und C. Möller (Hrsg.), Ars iuris. Festschrift für Okko Behrends, Göttingen 2009, S. $421 \mathrm{ff}$.

10 M. F. Cursi, Modelle objektiver Haftung im Deliktsrecht: Das schwerwiegende Erbe des römischen Rechts, SZ 132 (2015), S. 362 (379) stellt bei der Noxalhaftung heraus, dass es sich um eine Haftung aus Herrschaft oder nach der „Logik der Macht" (Branca) handele.

11 Zum Ausgangspunkt der Rechtsverletzung im Deliktsrecht, nicht der Autonomie des Individuums wie im Vertragsrecht s. A. Bürge, Die Entstehung und Begründung der Gefährdungshaftung im 19. Jahrhundert und ihr Verhältnis zur Verschuldenshaftung - Eine Skizze, in: A. Heldrich, J. Prölss, I. Koller u.a. (Hrsg.), Festschrift für Claus-Wilhelm Canaris zum 70. Geburtstag, München 2007, S. 59 (65). J.-E. Schirmer, Rechtsfähige Roboter? JZ 2016, S. 660 ff. plädiert für ein funktionales Verständnis der Rechtsfähigkeit, das den Zweck in den Mittelpunkt stellt. 


\section{B. Zur geschichtlichen Entwicklung und zu den verschiedenen Konzeptionen im römischen Recht}

\section{XII-Tafel-Zeit und Ausgangslage}

Schon in der ersten Kodifikation römischen Rechts aus der Mitte des 5. Jahrhunderts v. Chr., den XII Tafeln, ist für verschiedene Tatbestände der Personenverletzung (iniuria) eine Haftung geregelt. ${ }^{12}$ Bei Körperverletzung durch Knochenbruch sind Bußsätze festgelegt, die der Täter an das Opfer zu entrichten hat, außerdem bei einer Beleidigung, die als iniuria erfasst wird. Grundlage für die Regulierung von Verstümmelungen sind Sühneabkommen, pacta, die als Ersatz für die Talion gelten. Es handelt sich um ein Privatstrafrecht, für das es noch keine von Staats wegen betriebene Strafverfolgung als Parallele gibt. Verschiedene weitere Schäden sind in den XII Tafeln sanktioniert. So gibt es eine Klage wegen Weideschadens, die den Eigentümer des auf fremdem Grundstück weidenden Viehs haftbar macht, actio de pastu pecoris, oder auch eine Klage wegen anderweitigen Tierschadens, actio de pauperie. ${ }^{13}$

Welche Ausgangssituation kann man für die weitere Entwicklung der Haftungsthematik beschreiben? Bei deliktischen Schädigungen ist der Schädiger bzw. der Eigentümer des schadenstiftenden Akteurs (oder des schadenstiftenden Gegenstandes) zu einem Ausgleich oder zu einer Bußzahlung verpflichtet. Grundsätzlich bedarf es dazu einer gesetzlichen Regelung. Im römischen Recht findet man unterschiedliche Lehren der Gesetzesinterpretation. ${ }^{14}$ Im Deliktsrecht lässt sich die prinzipielle Argumentation dahingehend zusammenfassen, dass die gesetzlichen Regelungen zum Ziel haben, die Vorgabe zu konkretisieren, dass niemand einen ande-

12 M. Kaser, Römisches Privatrecht, 1. Abschnitt, 2. Aufl., München 1971, S. 155 ff.

13 S. dazu M. Kaser/R. Knütel, Römisches Privatrecht (Fn. 7), § 50 II. 4. b), S. 298 Rn. 14. Eine Klage wegen Verzauberns der Feldfrucht, wenn auf dem Nachbargrundstück unter verdächtigen Umständen die Ernte ausbleibt, soll hier nicht weiter thematisiert werden. Als sakrale Verbrechen von Kaser, Römisches Privatrecht (Fn. 12), S. 155 eingeordnet - entgegen Kunkel, der wohl eine private Bestrafung annimmt, so Kaser (Fn. 12).

14 S. dazu O. Behrends, Gesetz und Sprache. Das römische Gesetz unter dem Einfluss der hellenistischen Philosophie, in: O. Behrends und W. Sellert (Hrsg.), Nomos und Gesetz. Ursprünge und Wirkungen des griechischen Gesetzesdenkens, Göttingen 1995, S. 135, für die lex Aquilia insbes. S. 241 ff. (= Institut und Prinzip Band 1, Göttingen 2004, S. 91, insbes. S. 215 ff.) 
ren verletzen soll, während die institutionelle Richtung eine Verantwortlichkeit nur unter klar und präzise festgelegten Bedingungen anerkennt.

\section{Die lex Aquilia und ihre unterschiedlichen Interpretationen}

Das Zentrum des römischen Deliktsrechts ist die lex Aquilia. Nach römischer Überlieferung ist mit diesem Plebiszit im Jahr 286 v. Chr. eine wesentliche Neuerung für das Deliktsrecht zu verzeichnen. Die lex Aquilia wurde von dem Volkstribunen Aquilius vor der Volksversammlung der Plebejer eingebracht und von dieser mit Gültigkeit für alle römischen Bürger beschlossen. Sie regelte in drei Kapiteln den Schadensersatz in Fällen der Sachzerstörung und der Sachbeschädigung. ${ }^{15}$ Innovativ war die Gestaltung der Rechtsfolge, nämlich eine Schadensbestimmung nach dem Höchstwert der zerstörten Sache im zurückliegenden Jahr bzw. im Fall einer Beschädigung im zurückliegenden Monat. Diese Schadensfeststellung bedeutete gegenüber den festen Bußsätzen der XII Tafeln eine am Sachwert orientierte Flexibilisierung der Haftung, enthielt aber auch ein pönales Element. Dieses wurde verstärkt, wenn der Täter den Vorwurf zu Unrecht bestritt. War ein Prozess erforderlich, den der Geschädigte gewann, verdoppelte sich die Urteilssumme.

Für die Haftungsthematik noch bedeutsamer ist die Voraussetzung, die mit dem Wort iniuria bezeichnet wurde. Der Tatbestand musste in widerrechtlicher Weise verwirklicht worden sein, um die Haftung auszulösen.

Für welche deliktischen Handlungen sah die lex Aquilia eine Haftung vor? Im ersten Kapitel war die Tötung von fremden Sklaven und Sklavinnen sowie von vierfüßigen Herdentieren erfasst. Das zweite Kapitel sah einen Schadenersatz bei Forderungsvernichtung vor. Das dritte Kapitel erfasste die Sachbeschädigung durch konkret aufgelistete Handlungsweisen, nämlich urere, frangere, rumpere, also brennen, brechen oder reißen.

Für die Interpretation durch verschiedene Juristen lassen sich charakteristische Merkmale feststellen. Von dem vorklassischen Juristen Quintus

15 Eine ausführliche Darstellung mit zahlreichen rechtsvergleichenden Hinweisen findet man bei $R$. Zimmermann, The Law of Obligations. Roman Foundations of the Civilian Tradition, Cape Town, München 1992, S. 953 ff. Vgl. auch H. Hausmaninger, Das Schadensersatzrecht der lex Aquilia, 5. Aufl., Wien 1996. 
Mucius Scaevola, Konsul des Jahres 95 v. Chr., ${ }^{16}$ sind in späteren Juristenschriften Entscheidungen überliefert, die ein sehr weit gefasstes Verständnis von culpa, Verschulden, belegen. Die culpa steht in dem Baumschneiderfall, der im Folgenden wiedergegeben ist, als Begriff für die Grundlage der Klage - posse de culpa agi-, die eindeutig als eine actio legis Aquiliae einzuordnen ist. Im Wortlaut des Gesetzes findet sie ihren Anker in dem Begriff iniuria. Die Voraussetzung der Widerrechtlichkeit wird interpretatorisch mit der culpa angereichert. So ist die Tötung eines Sklaven durch einen beim Ausschneiden eines Baumes heruntergeworfenen Ast dem Baumausschneider, dem putator, zuzurechnen, weil er einen Warnruf unterlassen hat. Einen culpa-Vorwurf zieht sich derjenige zu, der nicht die Sorgfalt einsetzt, die ein umsichtig Handelnder an den Tag legen würde. ${ }^{17}$

\section{9,2,31 Paulus libro decimo ad Sabinum}

Si putator ex arbore ramum cum deiceret vel machinarius hominem praetereuntem occidit, ita tenetur, si is in publicum decidat nec ille proclamavit, ut casus eius evitari possit. sed Mucius etiam dixit, si in privato idem accidisset, posse de culpa agi: culpam autem esse, [quod cum] <cum quod $>$ a diligente provideri [poterit] < potuerit>, non esset provisum aut tum denuntiatum esset, cum periculum evitari non possit. secundum quam rationem non multum refert, per publicum an per privatum iter fieret, cum plerumque per privata loca volgo iter fiat. quod si nullum iter erit, dolum dumtaxat praestare debet, ne immittat in eum, quem viderit transeuntem: nam culpa ab eo exigenda non est, cum divinare non potuerit, an per eum locum aliquis transiturus sit.

Paulus im 10. Buch seines Kommentars zum Zivilrecht des Sabinus

Hat jemand, der beim Baumausschneiden einen Ast heruntergeworfen hat, oder der auf einem Gerüst arbeitete, einen vorübergehenden Sklaven getötet, so haftet er, vorausgesetzt, dass der Gegenstand auf einen öffentlichen Weg gefallen ist und er nicht vorher so laut gerufen hat, dass der Unfall des Sklaven hätte vermieden werden können. Aber Mucius hat gesagt, es könne auch dann wegen Verschuldens Klage erhoben werden, wenn sich dasselbe auf einem privaten Grundstück zugetragen hätte. Denn Verschulden liege [schon immer dann] vor, wenn Vorkehrungen nicht getroffen wurden, obwohl sie von einem umsichtigen Menschen getroffen werden konnten, oder die Warnung erst erfolgte, als sich die Gefahr nicht mehr vermeiden ließ. Nach diesem Grundsatz macht es keinen großen Unterschied, ob der Weg über öffentliche oder private Grundstücke führt, da ein allgemein benutzter Weg sehr oft über

16 O. Behrends, Art. Quintus Mucius Scaevola, in: Juristen, M. Stolleis (Hrsg.), München 1995 , S. $444 \mathrm{f}$.

17 C. Möller, Via publica und via privata im römischen Deliktsrecht (Fn. 9), S. 421 (436 ff.). So auch im Bärengrubenfall (S. 440 ff.) und im Stutenhetzfall. Zu letzterem s. insbes. die Musterexegese von O. Behrends, Römischrechtliche Exegese: Das deliktische Haftungssystem der lex Aquilia, JuS 1985, S. 878 ff. 
Privatgrundstücke führt. Gab es aber keinen Weg, muss der Täter jedenfalls für Vorsatz haften, damit er nicht etwa auf jemanden wirft, den er vorübergehen sieht. Ein Einstehen für Fahrlässigkeit [das heißt für Unterlassen der Sorgfaltsmaßnahmen] kann man nämlich [in einem solchen Fall] nicht von ihm verlangen, weil er nicht ahnen konnte, dass jemand gerade an dieser Stelle vorübergehen werde. ${ }^{18}$

Diese weite Formel, wonach alles hätte beachtet werden müssen, was von einem umsichtigen Menschen, einem diligens, beachtet worden wäre, ist in die Definition von § 276 Abs. 2 BGB zur Bestimmung der Fahrlässigkeit eingegangen. Bei Quintus Mucius liegt ihr ein Verständnis zugrunde, das jeden Menschen in die Pflicht nimmt, auf die Belange seiner Mitmenschen Rücksicht zu nehmen.

Die eben herausgestellte Definition der culpa von Quintus Mucius ist prinzipieller Natur. ${ }^{19}$ Daher spielt die Örtlichkeit, wo der Schadensfall sich ereignet, gerade keine Rolle. Eine Pflicht zur Rücksichtnahme und zum umsichtigen Handeln besteht überall. Diese Ansicht des Quintus Mucius teilen die späteren Juristen nicht. Sabinus und Paulus ${ }^{20}$ differenzieren nach dem Umfeld des Geschehens. Dort, wo eine Straße oder ein öffentlich genutzter Weg vorbeiführt, muss die diligentia beachtet werden. Auf einem Privatgrundstück, wo kein Weg unter dem Baum entlangführt, ist dies nicht der Fall. Diese Herangehensweise ist auch für die Speerwerferfälle überliefert, bei denen ein Sklave durch einen Übungswurf zu Tode gekommen ist. Stets wird danach unterschieden, ob der Speerwerfer sich auf einem Übungsgelände befand und sich daher beim Werfen nicht besonders vorsehen musste, oder ob das Speerwerfen an einem Ort praktiziert wurde, an dem man mit Publikumsverkehr rechnen musste. ${ }^{21}$ Eine Entscheidung, ob dem Schädiger ein größerer Vorwurf zu machen ist, oder ob dem Geschädigten ein höherer Anteil Eigenverschulden zukommt, war im römischen Recht erforderlich, weil eine Berücksichtigung von Mitverschulden

18 Übersetzung von O. Behrends/R. Knütel/B. Kupisch/H. H. Seiler, Corpus Iuris Civilis, Band II, Digesten 1-10, Heidelberg 1995.

19 C. Möller, Via publica und via privata im römischen Deliktsrecht (Fn. 9), S. 421 (438 ff.).

20 Zur Zuweisung des spätklassischen Juristen Paulus zur sabinianischen Rechtsschule s. C. Möller, Die Zuordnung von Ulpian und Paulus zu den kaiserzeitlichen Rechtsschulen, in: K. Muscheler (Hrsg.), Römische Jurisprudenz - Dogmatik, Überlieferung, Rezeption, Festschrift für Detlef Liebs zum 75. Geburtstag, Berlin 2011, S. $455 \mathrm{ff}$.

21 D. 9,2,9,4 Ulpianus libro octavo decimo ad edictum; Inst. 4,3,4. 
nicht vorgesehen war. ${ }^{22}$ Entweder haftete der Speerwerfer aufgrund einer actio legis Aquiliae oder dem Eigentümer des getöteten Sklaven stand keine Klage zu.

Der prinzipiellen Herangehensweise des Mucius und der Vorgabe, alle Sorgfalt zur Vermeidung einer Verletzung einzusetzen, entspricht einer der in der Fassung des Spätklassikers Ulpian überlieferten Rechtsgrundsätze, alterum non laedere: D. 1,1,10,1 libro primo regularum: Iuris praecepta sunt haec: honeste vivere, alterum non laedere, suum cuique tribuere. ${ }^{23}$

Ganz anders wird die lex Aquilia von den römischen Juristen interpretiert, denen die Rechtsordnung eine klar und bestimmt gefasste Sammlung von menschengemachten Normen ist. Rechtsstrukturen sind in Instituten festgelegt, möge dies in Gesetzen oder durch juristische Dogmatik geschehen sein. ${ }^{24}$ Im Deliktsrecht bedeutet das im Bereich der lex Aquilia eine Anknüpfung an Handlungen, durch die der gesetzlich erfasste Tatbestand verwirklicht wird. So interpretieren Servius Sulpicius und seine Schüler in der Mitte des 1. Jhs. v. Chr. die Schädigungshandlung der lex Aquilia als eine notwendig körperliche. ${ }^{25}$ Nur wer einem fremden Körper durch den eigenen Körper widerrechtlich einen Schaden zugefügt hat, haftet unmittelbar nach der lex Aquilia. Diese Vorgabe und ihre Konsequenz lassen sich anhand von D. 9,2,9 pr., § 2 und $\S 3$ nachvollziehen, einer Quelle, die aus Ulpians Ediktskommentar stammt. ${ }^{26}$ Nach dem augusteischen Juristen

22 H. Hausmaninger, Das Schadensersatzrecht der lex Aquilia (Fn. 15), S. 29.

23 Die Gebote des Rechts sind folgende: Ehrenhaft leben, niemanden verletzen, jedem das Seine gewähren. Übersetzung aus Behrends/Knütel/Kupisch/Seiler, Corpus Iuris Civilis (Fn. 18).

24 O. Behrends, Art. Servius Sulpicius Rufus, in: M. Stolleis (Hrsg.), Juristen, München 1995 , S. $562 \mathrm{f}$.

25 O. Behrends, Gesetz und Sprache (Fn. 14), S. 245 f. (= S. 219 f.)

26 D. 9,2,9 pr. Ulpianus libro octavo decimo ad edictum: Item si obstetrix medicamentum dederit et inde mulier perierit, Labeo distinguit, ut, si quidem suis manibus supposuit, videatur occidisse: sin vero dedit, ut sibi mulier offerret, in factum actionem dandam, quae sententia vera est: magis enim causam mortis praestitit quam occidit. § 2 Si quis hominem fame necaverit, in factum actione teneri Neratius ait. $\S 3$ Si servum meum equitantem concitato equo effeceris in flumen praecipitari atque ideo homo perierit, in factum esse dandam actionem Ofilius scribit: quemadmodum si servus meus ab alio in insidias deductus, ab alio esset occisus. Ulpian im 18. Buch zum Edikt: Weiter: hat eine Hebamme einer Sklavin eine Arznei gegeben, und ist diese daran gestorben, so unterscheidet Labeo: Hat die Hebamme die Arznei eigenhändig verabreicht, hat sie getötet. Hat die Hebamme aber die Arznei so gegeben, dass die Sklavin sie selbst einzunehmen hatte, dann muss eine auf den Sachverhalt zugeschnittene Klage erteilt werden. Diese Meinung ist 
Labeo haftet die Hebamme für den Tod einer Sklavin nur dann unmittelbar nach der lex Aquilia, wenn sie ihr die tödliche Arznei eigenhändig verabreicht hat, nicht dann, wenn die Sklavin es war, welche die bereitgestellte Arznei selbst eingenommen hat. Neraz, ein Schulhaupt der prokulianischen Rechtsschule Ende des 1. Jhs. n. Chr., behandelt den Fall entsprechend, in dem ein fremder Sklave durch Verhungern-Lassen getötet wird. Ofilius, ein Schüler des Servius Sulpicius, entscheidet einen weiteren Fall, der nicht direkt von der lex Aquilia erfasst wird. Gemeinsam ist diesen Fällen, dass der deliktisch Handelnde lediglich die Ursache für den Tod setzt, die causa mortis. ${ }^{27}$ Daher muss Rechtsschutz auf anderem Wege gesucht werden, nämlich mit Hilfe des Prätors, der als Gerichtsmagistrat über die Kompetenz verfügt, eine Klage zu schaffen, ein iudicium zu gewähren, und zwar eine auf den typisierten Sachverhalt zugeschnittene Klage, eine actio in factum..$^{28}$

Scheinen dies vielleicht auf den ersten Blick lediglich Besonderheiten des römischen Prozessrechts zu sein, ${ }^{29}$ so wird der auch unter pragmatischen Gesichtspunkten vorhandene Unterschied der Auffassungen gerade in der Entscheidung des Mucius im Baumschneider-Fall erkennbar. Hier würde nach der neueren Lehre des Servius eine Haftung ausscheiden, da es an einer unmittelbaren Zufügung des Schadens fehlt. Die culpa ist nicht mehr als Quelle von Sorgfalts- oder Verkehrssicherungspflichten akzeptiert und erweitert die Haftung daher nicht. Sie wird bei diesen Juristen zu einem nahezu nutzlosen Instrument, weil man eine unmittelbare körperli-

richtig. Denn die Hebamme hat nur die Ursache für den Tod gesetzt und nicht getötet. $§ 2$ Hat jemand einen Sklaven dadurch getötet, dass er ihn verhungern ließ, so haftet er nach Neraz mit einer auf den Sachverhalt zugeschnittenen Klage. § 3 Hast du das Pferd, auf dem mein Sklave ritt, scheu gemacht und dadurch bewirkt, dass er kopfüber in den Fluss gestürzt ist, und ist der Sklave dadurch umgekommen, so muss, wie Ofilius schreibt, eine auf den Sachverhalt zugeschnittene Klage erteilt werden; genauso wie wenn mein Sklave von jemandem in einen Hinterhalt gelockt und dort von einem anderen getötet worden wäre. Übersetzung aus Behrends/Knütel/Kupisch/Seiler, Corpus Iuris Civilis (Fn. 18).

27 Zum Zusammenhang mit dem Auftreten der actio in factum s. D. Nörr, Causa mortis, München 1986, S. 139 ff. und zu den Juristen, die diese Argumentationsfigur einsetzten auch S. $160 \mathrm{ff}$. und insoweit zustimmend die Besprechung von O. Behrends, Gnomon 61 (1989), S. 685 (692), weiterführend S. 697 ff.

28 O. Behrends, Gesetz und Sprache (Fn. 14), S. 247 f. (= S. 221 f.).

29 Unter diesem Aspekt beleuchtet die Haftung nach der lex Aquilia insbesondere A. Bürge, Römisches Privatrecht. Rechtsdenken und gesellschaftliche Verankerung, Darmstadt 1999, S. 18 ff. 
che Schädigung durch den putator nicht annehmen kann und eine Haftung für das Unterlassen einer Warnung oder einer Absicherung nicht in Betracht kommt. Die Zurechnung wird schon auf der Tatbestandsebene vorentschieden und scheitert häufig. Eine $»$ Filterfunktion $\aleph^{30}$ hat die culpa in diesem System nur noch für die Schuldfähigkeit. ${ }^{31}$ Eine actio in factum ist für diese Fälle nicht belegt.

In Kurzfassung kann man ein Konzept tatbestandlich präziser, auf die aktive Begehung von Delikten gerichteter Schadensersatzhaftung erkennen, dass sich ganz grundlegend von einem naturrechtlich-prinzipiellen Ansatz unterscheidet, dem zufolge die Anforderungen an ein sorgfältiges und rücksichtsvolles Verhalten auch eine Haftung bei Unterlassen tragen. Eine Haftungserweiterung konnte nach diesem Konzept nur durch neue Gesetze oder prätorische Rechtsbehelfe zustandekommen. Dies geschah durch neue Straftatbestände, aber insbesondere durch die Gewährung von auf den typisierten Sachverhalt zugeschnittenen Klagen, actiones in factum, und durch die Einführung der Gefährdungshaftung für bestimmte Situationen.

\section{Gefährdungshaftung im römischen Recht am Beispiel der actio de deiectis vel effusis}

Ansätze für eine Gefährdungshaftung erkennt man schon in den XII-Tafeln, zum Beispiel beim Einstehen-Müssen für Weideschaden und für Tierschaden. Einen neuen Zuschnitt bekommt diese verschuldensunabhängige Haftung durch die Aufnahme von Rechtsbehelfen ins Edikt. Es handelt sich um einen vom Prätor kraft seiner Amtsgewalt gewährten Schutz, der insbesondere die Gewährleistung der Sicherheit des Verkehrs auf öffentlichen Straßen und Wegen zum Ziel hat. Das Edikt de deiectis vel effusis verpflichtet den Bewohner eines Hauses zu Schadensersatz, wenn aus diesem Haus etwas hinausgeworfen oder hinausgegossen wurde, das einen Schaden verursacht hat. Nicht der Schädiger muss ermittelt werden, son-

30 O. Behrends, Römischrechtliche Exegese (Fn. 17), S. 878 (883).

31 Zur einerseits haftungserweiternden Rolle der culpa und ihrer andererseits haftungsausschließenden Funktion N. Jansen, Die Struktur des Haftungsrechts, Tübingen 2003, S. $252 \mathrm{ff}$. 
dern der Wohnungsinhaber (habitator) wird haftbar gemacht, wie man dem Ediktszitat im Kommentar Ulpians entnehmen kann. ${ }^{32}$

Die Einführung dieses prätorischen Rechtsschutzes findet erst dann eine vollständige Erklärung, wenn man den konzeptionellen Wandel im Deliktsrecht wahrnimmt, der mit der restriktiven Interpretation der lex Aquilia durch Servius und seine Schüler eingetreten war. Eine Haftung in den angesprochenen Fällen hätte wegen einer Missachtung der Verkehrssicherungspflicht oder wegen mangelnder Vorkehrungen gegenüber Gästen der Wohnung nach Quintus Mucius auch auf der Grundlage der aquilischen Regelung angenommen werden können. Doch war nach der neuen Interpretation eine unmittelbare körperliche Schadenszufügung in Verbindung mit dem Verschuldenserfordernis so strikt auf den handgreiflich Schädigenden bezogen, dass eine Klage aus dem Gesetz gegen den Wohnungsbesitzer nicht gewährt werden konnte.

Diese Konsequenz der neuen Interpretation wurde vom Prätor korrigiert, indem er gestützt auf die naturalis aequitas einen tatbestandlich konkreten Rechtsschutz durch ediktale Klagen schuf. Der Rechtsschutzauslöser ist ebenso neu bestimmt wie die Grundlage der Klage im imperi$u m$ des Gerichtsmagistraten. Auslöser des Rechtsschutzes ist eine Unsicherheit auf Verkehrswegen, Ziel der Regelung ist die Sicherheit derselben. ${ }^{33}$ Dieser Zweck leitet die Regelung der Haftung für Hinausgeworfenes und Hinausgegossenes. In seinem Ediktskommentar hat Ulpian ein Lob festgehalten. Die Anordnung des Prätors sei höchst zweckmäßig, da es im öffentlichen Interesse liege, dass man ohne Furcht und Gefahr auf den Straßen gehen kann. ${ }^{34}$

Die Juristen berücksichtigen den Zweck des Schutzes öffentlicher Wege auch bei der Anwendung dieser Gefährdungshaftung. Die Auslegung führt zu einer Ausdehnung auch auf solche Wege, die zwar als Privatwege ein-

32 D. 9,3,1 pr. Ulpianus lb. 23 ad ed.: Praetor ait de his, qui deiecerint vel effuderint: "Unde in eum locum, quo volgo iter fiet vel in quo consistetur, deiectum vel effusum quid erit, quantum ex ea re damnum datum factumve erit, in eum, qui ibi habitaverit, in duplum iudicium dabo. ..." Vgl. dazu J. D. Harke, Sklavenhalterhaftung in Rom, in diesem Band, S. $101 \mathrm{f}$.

33 Die Verhältnisse sind anschaulich geschildert bei $R$. Zimmermann, Effusum vel deiectum, in: D. Medicus, H.-J. Mertens, K. W. Nörr, W. Zöllner (Hrsg.), Festschrift für Hermann Lange, Köln 1992, S. 301 ff.

34 D. 9,3,1,1 Ulpianus libro vicensimo tertio ad edictum: Summa cum utilitate id praetorem edixisse nemo est qui neget: publice enim utile est sine metu et periculo per itinera commeari. 
geordnet, aber gewöhnlich öffentlich genutzt werden. Für alle Orte (loca), über die man gewöhnlich geht, müsse dieselbe Sicherheit geboten werden. ${ }^{35}$ Es ist interessant zu sehen, dass die konkrete Nutzung hier haftungserweiternd wirkt, während sie bei dem putator-Fall des Mucius von den späteren Juristen Sabinus und Paulus gegenüber dem allgemeinen Rücksichtnahmeprinzip einschränkend eingesetzt wird. Die Verkehrssicherungspflichten bestehen danach nur dort, wo auch mit Betrieb auf einem Weg, sei er öffentlich oder privat, zu rechnen ist. ${ }^{36}$

Dieser Schutzzweck der Sicherheit von Verkehrswegen wird durch weitere prätorische Klagen verfolgt, so für Gefährdungen durch Gegenstände, die auf einem Wetterdach oder einem Vorbau aufgestellt waren, ${ }^{37}$ aber auch für Schäden durch das Halten wilder Tiere in der Nähe von Straßen. Verursachen diese einen Schaden, so haben die kurulischen Ädile eine $a c$ tio de feris in ihr Edikt aufgenommen, bei welcher der Schutz eines gewöhnlich dort genutzten Weges einen Schadensersatzanspruch trägt. ${ }^{38}$ Verschulden ist für diese Haftung nicht erforderlich. Man kann vielmehr von einer Gefährdungshaftung sprechen. ${ }^{39}$

Diese Zuschreibung einer Haftung ist möglicherweise dadurch mitveranlasst gewesen, dass im römischen Recht keine anteilige Berücksichtigung eines Mitverschuldens möglich war, sondern ein Alles-oder-NichtsPrinzip galt. Es musste stets entschieden werden, wer im höheren Maße

35 D. 9,3,1,2 Ulpianus lb. 23 ad ed.: Parvi autem interesse debet, utrum publicus locus sit an vero privatus, dummodo per eum volgo iter fiat, quia iter facientibus prospicitur, non publicis viis studetur: semper enim ea loca, per quae volgo iter solet fieri, eandem securitatem debent habere. ... S. auch J. D. Harke, Sklavenhalterhaftung (Fn. 32), S. 103.

36 M. F. Cursi (Fn. 10), S. 362 (383 f.) sieht einen Zusammenhang mit der urbanen und kommerziellen Expansion Roms. Prätor und Jurisprudenz hätten das Interesse am Schadensausgleich vor das Prinzip subjektiver Verantwortung gerückt. Mechanismen objektiver Haftung hätten dieses Prinzip ergänzt. Diese Analyse scheint mir zutreffend, doch keineswegs erschöpfend. Die dogmatische Alternative zeigt, dass die bewusste Bevorzugung eines Haftungsmodells den Ergänzungsbedarf auslöste. Das Nebeneinander der Regelungen und der Stellungnahmen der Juristen verdunkelte die unterschiedlichen systematischen Konzepte.

37 D. 9,3,5,6 Ulpianus lb. 23 ad ed.

38 D. 21,1,40,1 und 42 Ulpianus lb. 2 ad ed. aed. und dazu C. Möller (Fn. 9), S. 421 (442 f.) sowie J. D. Harke, Sklavenhalterhaftung (Fn. 32), S. 101 Fn. 9. Die Auslegung der ediktalen Bestimmungen, wie sie in den Fragmenten der Digesten nachgelesen werden kann, ist deutlich am Zweck des Edikts orientiert.

39 R. Zimmermann, Effusum vel deiectum (Fn. 33), S. 301 ff.; M. F. Cursi (Fn. 10), S. 362 (379 ff.). 
die Verantwortung für einen Schaden zu tragen hatte. Von einer solchen Zuschreibung sollte der Nutzer öffentlich begangener Wege grundsätzlich freigestellt sein. Die Juristen akzeptierten in diesen Fällen also eine Haftung ohne ein Verschulden im Sinne einer persönlichen Vorwerfbarkeit. ${ }^{40}$

\section{Andere Akteure und Übernahmeverschulden}

Die Einschaltung von Hilfspersonen hat die Frage nach den Voraussetzungen der Haftung ihrer Eigentümer oder Hausväter, allgemein ihrer Gewalthaber, ausgelöst. Ich beschränke mich auch hier auf Fragen der deliktischen Haftung. ${ }^{41}$ Es ist zu beachten, dass diese Haftung mangels eigenen Vermögens der Hilfspersonen zwar immer den Gewalthaber treffen muss, damit der Geschädigte einen Ersatz erhält, dass diese Haftung aber je nach dem eigenen Anteil des Gewalthabers unterschiedlich ausgestaltet ist. ${ }^{42} \mathrm{Im}$ Rahmen der Noxalhaftung ist die Abgrenzung eines Eigenverschuldens von einem Verschulden der Hilfspersonen oder einer Zurechenbarkeit des unfallstiftenden Verhaltens von Nutztieren ein wichtiges Element der Vergewisserung über Unterscheidungen bei der deliktsrechtlichen Haftung. ${ }^{43}$

Als andere Akteure sollen Maultiere in den Mittelpunkt gestellt werden, die beim Erklimmen des kapitolinischen Hügels an einem Unfall beteiligt waren. Folgender Sachverhalt wird in den Digesten aus einem Werk des spätrepublikanischen Juristen Alfenus überliefert:

D. 9,2,52,2 Alfenus lb. 2 digestorum

In clivo Capitolino duo plostra onusta mulae ducebant: prioris plostri muliones conversum plostrum sublevabant, quo facile mulae ducerent: [inter] superius plostrum cessim ire coepit et cum muliones, qui inter duo plostra fuerunt, e medio exissent, posterius plostrum a priore percussum retro redierat

40 Doch findet man auch das Argument, dass die Haftung deswegen angemessen sei, weil bei dem Wohnungseigentümer, anders als beim Hauseigentümer culpa vorliege. D. 9,3,1,4 Ulpianus 1b. 23 ad ed. und dazu M. F. Cursi (Fn. 10), S. 362 (385, Fn. 80). Sogleich wird aber von Ulpian klärend festgehalten, dass die actio de effusis vel deiectis kein Verschuldenserfordernis kenne und sich insoweit von der lex Aquilia unterscheide. S. auch J. D. Harke, Sklavenhalterhaftung (Fn. 32), S. 105 f.

41 Zur Haftung für Hilfspersonen im römischen Recht siehe den umfangreichen Aufsatz von $R$. Knütel, Die Haftung für Hilfspersonen im römischen Recht, SZ 100 (1983), S. $340 \mathrm{ff}$.

42 S. dazu den Beitrag von J. D. Harke, Sklavenhalterhaftung in Rom, in diesem Band S. $97 \mathrm{ff}$.

43 Ebenso und eingehend zu den einzelnen Fragen M. F. Cursi (Fn. 10), S. 362 ff. 
et puerum cuiusdam obtriverat: dominus pueri consulebat, cum quo se agere oporteret, respondi in causa ius esse positum: nam si muliones, qui superius plostrum sustinuissent, sua sponte se subduxissent et ideo factum esset, ut mulae plostrum retinere non possint atque onere ipso retraherentur, cum domino mularum nullam esse actionem, cum hominibus, qui conversum plostrum sustinuissent, lege Aquilia agi posse: nam nihilo minus eum damnum dare, qui quod sustineret mitteret sua voluntate, ut id aliquem feriret: veluti si quis asellum cum agitasset non retinuisset, aeque si quis ex manu telum aut aliud quid immisisset, damnum iniuria daret. sed si mulae, quia aliquid [reformidassent] <recessissent $>$ et muliones timore permoti, ne opprimerentur, plostrum reliquissent, cum hominibus actionem nullam esse, cum domino mularum esse. quod si neque mulae neque homines in causa essent, sed mulae retinere onus nequissent aut cum coniterentur lapsae concidissent et ideo plostrum cessim redisset atque hi [quo] < quod $>$ conversum fuisset onus sustinere nequissent, neque cum domino mularum neque cum hominibus esse actionem. illud quidem [certe] <certum esse $>$, quoquo modo res se haberet, cum domino posteriorum mularum agi non posse, quoniam non sua sponte, sed percussae retro redissent. ${ }^{44}$

44 Alfen im 2. Buch seiner Digesten: Maultiere zogen zwei beladene Lastkarren den kapitolinischen Hügel hinauf. Die Maultiertreiber des vorderen Karrens versuchten, den nach hinten abkippenden Karren anzuheben, damit die Maultiere ihn leichter ziehen konnten. Der vordere Karren begann nach rückwärts zu rollen, und als die Maultiertreiber, die sich zwischen den beiden Karren befanden, aus dem Zwischenraum zur Seite gesprungen waren, wurde der hintere Karren vom vorderen angestoßen, rollte nach rückwärts und erdrückte einen Sklavenjungen, der einem Dritten gehörte. Der Eigentümer des Sklavenjungen fragte an, wen er verklagen solle. Ich habe gutachtlich geantwortet, dass die Rechtslage von den näheren Umständen des Sachverhalts abhänge. Wenn nämlich die Maultiertreiber, die den vorderen Karren gestützt hatten, ohne Not ihren Platz verlassen hätten und es dadurch gekommen sei, dass die Maultiere den Karren nicht mehr halten konnten und durch das bloße Gewicht der Last nach hinten gezogen wurden, so habe der Eigentümer des Sklavenjungen zwar gegen den Eigentümer der Maultiere keine Klage, wohl aber könnten die Leute, die den nach hinten abkippenden Karren gestützt hatten, nach der lex Aquilia verklagt werden. Denn auch der fügt [durch sein Handeln] Schaden zu, der aus eigenem Willensentschluss etwas nur loslässt, das er hält, so dass jemand davon getroffen wird. Wer zum Beispiel einen jungen Esel, nachdem er ihn angetrieben hatte, nicht mehr zurückhalten konnte, fügt widerrechtlich Schaden zu, ebenso wie derjenige, der ein Wurfgeschoß oder einen anderen Gegenstand seiner Hand entfahren lässt. Wenn dagegen die Maultiere, weil sie vor irgendetwas gescheut hätten, zurückgewichen seien und die Maultiertreiber aus Furcht, erdrückt zu werden, ihren Platz hinter dem Karren verlassen hätten, so habe der Eigentümer des Sklavenjungen zwar gegen die Maultiertreiber keine Klage, wohl aber gegen den Eigentümer der Maultiere. Entsprächen aber weder die Maultiere noch die Leute insoweit den Klagevoraussetzungen, hätten vielmehr die Maultiere die Last einfach nicht halten können oder seien sie, während sie sich mit 
Maultiere ziehen zwei schwerbeladene Lastkarren den Hügel hinauf. Die Maultiertreiber versuchen, die Last zu erleichtern, treten zwischen die beiden Karren und heben den ersten hinten an. Der gewünschte Effekt bleibt aus, der vordere Karren beginnt vielmehr rückwärts zu rollen. Die Maultiertreiber springen zur Seite, der vordere Wagen stößt den hinteren an, der nun nach hinten rollt und einen Sklavenjungen erdrückt. Dieser Sklavenjunge gehörte einem Dritten. So löste der von Alfenus, einem Schüler des Servius, berichtete Fall ein Rechtsgutachten aus, da der Eigentümer des getöteten Sklavenjungen wissen wollte, gegen wen er Klage erheben könne.

Alfenus oder sein Lehrer Servius haben in dem Gutachten vor allem darauf hingewiesen, dass man den Sachverhalt noch näher aufklären müsse. Wenn nämlich die Maultiertreiber ohne Not ihren Platz verlassen hätten, dann könnten diese nach der lex Aquilia verklagt werden. ${ }^{45}$ Der Eigentümer der Maultiere aber könne nicht haftbar gemacht werden. Diese Entscheidung wird durch folgende Argumentation gestützt: auch wenn jemand aus eigenem Willensentschluss nur etwas loslässt, was er hält, so dass jemand getroffen wird, haftet er. Diese Argumentation soll erkennbar dem Einwand begegnen, dass keine unmittelbare körperliche Schädigung vorliegt. 46

Dieser möglichen Konkretisierung des Sachverhalts wird eine andere gegenübergestellt. Wenn nämlich die Maultiere vor irgendetwas gescheut hätten und deswegen zurückgewichen seien und die Maultiertreiber ( $m u$ liones) aus Furcht davor, erdrückt zu werden, ihren Platz verlassen hätten,

aller Kraft in die Riemen legten, ausgeglitten und niedergestürzt und sei der Karren deswegen rückwärts gerollt und hätten die Leute die Last nicht mehr halten können, weil der Karren nach hinten abgekippt war, so sei weder gegen den Eigentümer der Maultiere noch gegen die Maultiertreiber eine Klage gegeben. Das aber stehe jedenfalls fest, wie immer sich die Sache auch zugetragen habe, dass der Eigentümer der hinteren Maultiere nicht verklagt werden kann, weil diese nicht aus eigenem Antrieb, sondern infolge des Stoßes zurückgewichen sind. (Übersetzung aus: Behrends/Knütel/Kupisch/Seiler, Corpus Iuris Civilis (Fn. 18)).

45 Es muss sich also in diesem Text um freie Maultiertreiber handeln. Siehe zu den Arbeitsverhältnissen in diesem Wirtschaftszweig C. Möller, Die mercennarii in der römischen Arbeitswelt, SZ 110 (1993), S. 296 ff.

46 Als weiteres Beispiel wird ein junger Esel angeführt, den man angetrieben habe und dann nicht mehr hätte halten können, oder auch der Schaden, der durch ein Wurfgeschoss verursacht wird, das der Hand entfährt und nicht gezielt geschleudert wird. 
dann träfe die Haftung den Eigentümer der Maultiere. Die muliones wären in diesem Fall ohne culpa.

Es schließt sich eine weitere Überlegung an, die zeigt, dass Alfenus auch die Möglichkeit bedacht hat, dass keine Klage zu gewähren ist. Wenn nämlich die Maultiere die Last nicht hätten tragen können, obwohl sie alle Anstrengungen unternommen hatten, und auch die Maultiertreiber die Last einfach nicht hätten halten können, sei weder gegen den Eigentümer der Maultiere noch gegen die Maultiertreiber eine Klage gegeben. Diese recht restriktiv bestimmte Haftung lässt eine erhebliche Rechtsschutzlücke, weil eine zu schwere Beladung als ein Risiko betrachtet wird, dessen mögliche Konsequenzen vom Geschädigten zu tragen sind.

Ganz anders ist in der sabinianischen Rechtsschule bei dem Juristen Gaius in seinem Kommentar zum Provinzialedikt die Haftung von Maultiertreibern oder von Reitern eingeordnet worden.

D. 9,2,8,1 Gaius libro septimo ad edictum provinciale:

Mulionem quoque, si per imperitiam impetum mularum retinere non potuerit, si eae alienum hominem obtriverint, volgo dicitur culpae nomine teneri, idem dicitur et si propter infirmitatem sustinere mularum impetum non potuerit: nec videtur iniquum, si infirmitas culpae adnumeretur, cum affectare quisque non debeat, in quo vel intellegit vel intellegere debet infirmitatem suam alii periculosam futuram. idem iuris est in persona eius, qui impetum equi, quo vehebatur, propter imperitiam vel infirmitatem retinere non poterit. ${ }^{47}$

Eine Haftung wegen Verschuldens, wegen culpa, und damit nach der lex Aquilia befürwortet er, wenn ein Maultiertreiber aus Unerfahrenheit oder aus Mangel an Kraft nicht verhindern kann, dass ein fremder Sklave niedergetrampelt wird. Die Haftung trifft ihn, weil nach Gaius niemand eine

47 Gaius im 7. Buch zum Provinzialedikt: Auch ein Maultiertreiber haftet, wie man gewöhnlich sagt, wegen Verschuldens, wenn er durchgehende Maultiere aus Unerfahrenheit nicht zurückhalten kann und diese einen fremden Sklaven niedertrampeln. Dasselbe nimmt man auch an, wenn er aus Mangel an Kraft die durchgehenden Maultiere nicht zurückhalten konnte. Und es erscheint nicht unangemessen, wenn Mangel an Kraft dem Verschulden zugerechnet wird, weil niemand eine Aufgabe übernehmen soll, bei der, wie er erkennt oder erkennen muss, sein Mangel an Kraft für einen anderen gefährlich werden kann. Dasselbe gilt in dem Fall des Reiters, der das durchgehende Pferd infolge Unerfahrenheit oder mangels Kraft nicht zu zügeln vermag. (Übersetzung aus: Behrends/Knütel/Kupisch/Seiler, Corpus Iuris Civilis (Fn. 18)). 
Aufgabe übernehmen soll, bei der, wie er erkennt oder erkennen muss, sein Mangel an Kraft für einen anderen gefährlich werden kann. ${ }^{48}$

Zusammenfassend kann festgehalten werden, dass der Einsatz von Maultieren als Helfern im Straßenverkehr einem Haftungsregime unterliegt. Wenn eine Schädigung Dritter durch den Einsatz der Maultiere verursacht worden ist, haftet der Eigentümer der Maultiere. Ganz unterschiedlich ist allerdings die Reichweite der Sorgfaltspflichten bestimmt. Nach Servius / Alfenus gilt nur in der konkreten Situation die Maßgabe, alles zu tun, um eine Schädigung anderer zu vermeiden. In der Überlieferung des Gaius sind die Anforderungen strenger: er befürwortet eine Haftung schon dann, wenn die Übernahme einer Tätigkeit erfolgt, obwohl bei einer sorgfältigen Einschätzung der Möglichkeiten eine Gefahr für Dritte drohte. Die Anknüpfung für die Haftung ist also vorverlagert.

\section{Schlussfolgerungen}

Was hat nun das Maultier mit dem intelligenten Agenten zu tun? Welche konzeptionellen Linien können wir dem Ausflug in das römische Recht entnehmen?

Maultiere sind anders als intelligente Agenten nicht programmiert, aber eingeübt in typische Abläufe und zugleich lernfähig. Trotz eines hohen Grades von Zuverlässigkeit kommt es gelegentlich zu Abweichungen von einem optimalen Verhalten. Maultiere haben kein Vermögen. Sie werden von Menschen eingesetzt und sind einer menschlichen Steuerung zugänglich. Greift diese Steuerung ein, knüpfen die Haftungsregeln an das menschliche Verhalten an. Agiert das Maultier »autonom « in schadenstiftender Weise, kann eine Haftung aus dem Gesichtspunkt des Übernahmeverschuldens oder eine Gefährdungshaftung statuiert werden. Eine weitere Option bildet eine Ausweitung von Sorgfaltspflichten.

Es ergibt sich ein Bild von Haftungsoptionen, das dogmatisch eine große Nähe zu der gegenwärtigen Debatte aufweist. Für die Anknüpfung ist eine Vergewisserung über die Vorgaben im tatsächlichen Bereich notwen-

48 Ähnlich auch Ulpian, der eine Haftung bejaht, wenn sich jemand eine zu große Last aufgeladen hat. Er führt den Juristen Pegasus aus dem 1. Jh. n. Chr. mit dem Hinweis an, dass eine Haftung bei zu schwerer Beladung greife oder dann, wenn jemand zu unvorsichtig über eine schlüpfrige Stelle gegangen sei. D. 9,2,7,2 U1pianus lb. 18 ad ed. 
dig, da eine entscheidende Weichenstellung beim Einsatz intelligenter Agenten dort stattfindet, wo ein menschlicher Akteur während des Betriebs die Möglichkeit zum Eingreifen behält. Dass diese Konstellation noch auf Jahrzehnte im Straßenverkehr dominieren wird, ist trotz der verstärkten Anstrengungen von Google oder Apple, Autobauer für eine Kooperation bei der Inbetriebnahme selbstfahrender Autos zu gewinnen, ziemlich sicher. ${ }^{49}$ Es nährt auch, wie am 29.12.2015 in der F.A.Z. nachzulesen war, die Hoffnung der Fahrlehrer, dass ihr Berufsstand nicht überflüssig werden, sondern vielmehr einen Innovationsschub erleben wird, wenn der Fahrlehrer zu einem Technik-Coach wird. Den Fahrer trifft in diesen Fällen - immer nach Prüfung von Systemfehlern, die eine Verantwortlichkeit ausschließen oder mindern - ein eigener Anteil an der Haftung.

Wenn es sich um »echte« selbstgesteuerte Akteure handelt, so kann die Haftung daran anknüpfen, dass diese von den Herstellern und von den Betreibern zum Einsatz gebracht worden sind. Das Gefährdungspotential kann im Rahmen der deliktsrechtlichen Dogmatik dann entweder Grundlage für Verkehrssicherungspflichten und damit für eine Verschuldenshaftung nach §823 I BGB sein oder vom Gesetzgeber als Gefährdungshaftung spezifisch ausgestaltet werden. ${ }^{50}$ Zwischen diesen Optionen einer Ausdehnung von Verkehrssicherungspflichten und einer gesetzlichen Regelung einer roboterspezifisch ausgestalteten Gefährdungshaftung bewegen sich die aktuellen Vorschläge. ${ }^{51}$

49 J. Nida-Rümelin, Lasst die Hände am Steuer! F.A.S. Nr. 28 vom 17.7.2016, S. 22 hat dies auch als Konsequenz eines nicht auflösbaren philosophischen Problems bezeichnet. Das Abwägen der Gründe menschlichen Handelns lasse sich wegen seiner Komplexität nicht in einen Algorithmus übersetzen.

50 Zum Vorschlag für eine Roboterhaftung als Gefährdungshaftung s. J. Hanisch, Zivilrechtliche Haftungskonzepte für Robotik, in: E. Hilgendorf (Hrsg.), Robotik im Kontext von Recht und Moral, Baden-Baden 2014, S. 27, (54), zur konstruktiven Alternative (36).

51 Schirmer, Rechtsfähige Roboter? (Fn. 11), S. 660 (665) spricht sich für eine Gefährdungshaftung des Inhabers aus. Dies sei aufgrund der fremdnützigen Ausrichtung der Tätigkeit des Roboters angemessen. Eine eigene Haftung des Roboters scheitere, wie er überzeugend festhält, nicht daran, dass nicht-menschliche Akteure nicht haften könnten - das Gegenbeispiel bietet die Haftung juristischer Personen - oder dass dem Roboter ein eigenes Vermögen fehle - dieses Problem könnte durch einen Haftungsfond oder eine Versicherungslösung behoben werden. Im römischen Recht sind dafür die adjektizischen Klagen mit einem Zugriff auf das Vermögen des Gewalthabers geschaffen worden. Vgl. für einen ersten Eindruck $\mathrm{Ka}$ - 
Diese Modelle waren - wie gezeigt werden sollte - bereits dem römischen Recht bekannt. Sie existierten in einem nicht entschiedenen Wettstreit miteinander. Darin liegt ein Hauptgrund für die unterschiedlichen Konsequenzen, die in der Geschichte der Aneignung des römischen Rechts aus diesem Überlieferungsschatz gezogen worden sind. ${ }^{52}$ Die Klärung dieser Zusammenhänge ist für neue Regelungsfelder ebenso nützlich wie für das Verständnis unterschiedlicher Haftungssysteme in Europa und der europäisch beeinflussten Haftungssysteme in anderen Teilen der Welt.

ser/Knütel (Fn. 7), § 49. Doch würde eine solche Eigenhaftung der ausschließlich fremdnützigen Rolle des Roboters nicht gerecht.

52 A. Bürge, Entstehung und Begründung der Gefährdungshaftung, (Fn. 11), S. 59 ff. 